\title{
Influence of External Electric Field on Time of Nonlinear Optical Reorientational Effect in Nematics
}

\author{
W.K. BAJDECKI* AND M.A. KARPIERZ \\ Faculty of Physics, Warsaw University of Technology \\ Koszykowa 75, 00-662 Warsaw, Poland
}

\begin{abstract}
Nonlinear reorientation phenomena in nematic liquid crystals cause extremely large refractive index changes. However, this effect is relatively slow and the determination of the time necessary to appearing or disappearing of the nonlinear effect is an important issue. In this work we present measurements of the time of increasing the nematics reorientation induced by the light beam passing through the liquid crystalline layer. The influence of external low-frequency electric field suppressing reorientation is also reported. The obtained results describing relations between time and optical power of light as well as between time and external electric field intensity are in good agreement with theory.
\end{abstract}

PACS numbers: 42.65.-k, 42.70.Df

\section{Introduction}

Liquid crystals are well known anisotropic materials and their properties were analyzed in many books and papers [1,2]. In the last years the growing interest in new applications of liquid crystals is still observed. Nematic liquid crystals are also excellent media in nonlinear optics. They have the giant optical nonlinearity due to reorientational phenomenon, which is not observed in other types of materials. The main drawback in applications of reorientational nonlinearity in nematics is a relatively low time necessary to appearing and disappearing of the nonlinear effect. In this paper the detailed measurements of the relaxation time of appearing the nonlinearity is presented.

The reorientation nonlinearity can be changed by external low-frequency electric field which modifies the value of nonlinear refractive index and also the

* corresponding author; e-mail: bajdecki@if.pw.edu.pl 
time of appearing of the nonlinear effect. In this work we present experimental results of influence of electric field on observed nonlinearity.

\section{Theory}

The reorientation process is investigated in the nematic liquid crystalline cell with homeotropic alignment (Fig. 1). The director $n$, defined as the mean direction of molecules long axis, changes locally its direction due to both optical and electric external fields $[1,2]$. In the analyzed configuration electric fields

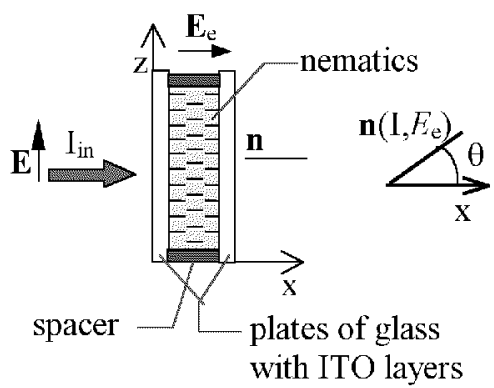

Fig. 1. Light propagation through the nematic film with applied electric field. Director $\boldsymbol{n}$ defines the average direction of long axis of the liquid crystal molecules.

and director at boundaries define one plane that allows to introduce an angle $\theta$ : $n=(\sin \theta, 0, \cos \theta)$, which describes the orientation of liquid crystals. The interactions between molecules as well as between molecules and external fields lead to appearing of new equilibrium state. After minimization of the total free energy from the Euler-Lagrange-Rayleigh equations the change of the orientation angle $\theta$ is obtained in the form $[1,2]$ :

$$
K \frac{\partial^{2} \theta}{\partial x^{2}}+\left(\frac{\varepsilon_{0} \Delta \varepsilon}{4} E^{2}-\frac{\varepsilon_{0} \Delta \varepsilon_{e}}{2} E_{\mathrm{e}}^{2}\right) \sin 2 \theta=\gamma \frac{\partial \theta}{\partial t}
$$

where $\Delta \varepsilon=n_{\mathrm{e}}^{2}-n_{\mathrm{o}}^{2}$ is the optical anisotropy described by extraordinary $n_{\mathrm{e}}$ and ordinary $n_{\circ}$ indices, $\Delta \varepsilon_{\mathrm{e}}=\varepsilon_{\|}-\varepsilon_{\perp}$ is the electric anisotropy described by parallel $\varepsilon_{\|}$and perpendicular $\varepsilon_{\perp}$ part of electric permittivity, $\varepsilon_{0}$ is the permittivity of the free space, $K$ is the elastic constant (in one-elastic constant approximation) resulting from the liquid crystalline elastic deformation, and $\gamma$ is the rotational viscosity of liquid crystals. The electrical fields are denoted as $E$ for optical field and $E_{\mathrm{e}}$ for low-frequency one.

Assuming small reorientation $\sin \theta \approx \theta$ in homeotropic strong anchoring alignment $\theta(x=0)=\theta(x=d)=0$, one can find the simplified solution in the form $\theta(x, t)=\theta_{0} \exp \left(t / \tau_{0}\right) \sin (\pi x / d)$, where the relaxation time $\tau_{0}$ is defined by

$$
\frac{\gamma}{\tau_{0}}=\frac{1}{2} \varepsilon_{0} \Delta \varepsilon E^{2}-\varepsilon_{0} \Delta \varepsilon_{\mathrm{e}} E \mathrm{e}^{2}-\frac{\pi^{2} K}{d^{2}} .
$$


The reorientation begins for $\tau_{0}>0$, which requires that optical field is larger than the threshold value

$$
E^{2} \geq \frac{2 \Delta \varepsilon_{\mathrm{e}}}{\Delta \varepsilon} E_{\mathrm{e}}^{2}+\frac{2 \pi^{2} K}{\varepsilon_{0} \Delta \varepsilon d^{2}}
$$

One can expect a similar relation to this described by Eq. (2) if the incident light has finite size dimension. Generally, the laser sources have the Gaussian-shape beam with the electric field intensity distribution in the form $E^{2}(r)=E^{2} \exp \left(-2 r^{2} / w_{0}^{2}\right)$, where $w_{0}$ is the beam waist. With small reorientation angle approximation, the steady-state distribution of the angle $\theta$ is now described by the relation $\theta(r, x, t)=$ $R(r, t) \sin (\pi x / d)$, which satisfies the strong anchoring conditions $(\theta=0$ for $x=0$ and $x=d$ ). The transversal profile of reorientation in one-constant approximation takes the form $[3-5]$ :

$$
\begin{aligned}
& K \frac{\partial^{2} R}{\partial r^{2}}+\frac{K}{r} \frac{\partial R}{\partial r}-\frac{K \pi^{2}}{d^{2}} R \\
& +\left[\frac{\varepsilon_{0} \Delta \varepsilon}{4} E^{2} \exp \left(-\frac{2 r^{2}}{w_{0}^{2}}\right)-\frac{\varepsilon_{0} \Delta \varepsilon_{\mathrm{e}}}{2} E_{\mathrm{e}}^{2}\right]\left(2 R-R^{3}\right)=\gamma \frac{\partial R}{\partial t}
\end{aligned}
$$

where $r$ is the radial coordinate. In a case of small reorientation $\left(R^{3} \ll R\right)$ the amplitude of transversal profile $R$ can be approximated by the Gaussian shape and the simplified solution in the form $R=R_{0} \exp (t / \tau) \exp \left(-r^{2} / w^{2}\right)$ can be assumed. The corresponding relaxation time $\tau$ is respectively defined by [6]:

$$
\frac{\gamma}{\tau}=\frac{1}{2} \varepsilon_{0} \Delta \varepsilon E^{2} \exp \left(-\frac{2 r^{2}}{w_{0}^{2}}\right)-\varepsilon_{0} \Delta \varepsilon_{\mathrm{e}} E_{\mathrm{e}}^{2}-\frac{K \pi^{2}}{d^{2}}-\frac{4 K}{d^{2}}\left(1-\frac{r^{2}}{w^{2}}\right) .
$$

The relaxation time $\tau$ corresponding to the finite size beam has a similar dependence on electric fields like relaxation time defined for plane wave $\tau_{0}$. Because the intensity of light beam is dependent on the transversal coordinate $r$, the time $\tau$ is also changing across the light beam. Additionally, the relaxation time is dependent on the width $w$ which is dependent on the light beam width $w_{0}$. This means that the time of increasing and decreasing the nonlinear reorientation in the light beam center depends on the light beam width.

\section{Experimental}

The theoretical prediction was checked in the experimental setup presented in Fig. 2a. As the light source we used semiconductor laser at wavelength $842 \mathrm{~nm}$, which was pig-tailed with a single mode fiber. After focalizing the beam waist was equal to $5.3 \mu \mathrm{m}$ and the focus was placed at the half of the cell thickness. As a liquid crystal we used 4-trans- $4^{\prime}$ - $n$-hexyl-cyclohexyl-isothiocyanatobenzene (6CHBT). The behavior of the nematic layer of thickness $60 \mu \mathrm{m}$ was observed simultaneously by the CCD camera and the detector with $2 \mathrm{~mm}$ aperture set in the 

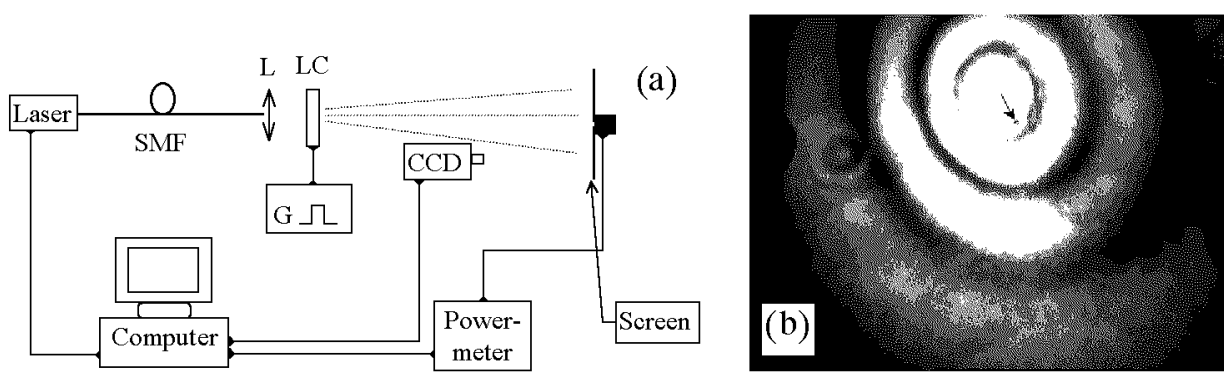

Fig. 2. Observation and detecting of nonlinear reorientation time of response of nematic molecules caused by external electric and optical fields: (a) experimental setup and (b) typical diffraction image with added black circle marking the position of detector.

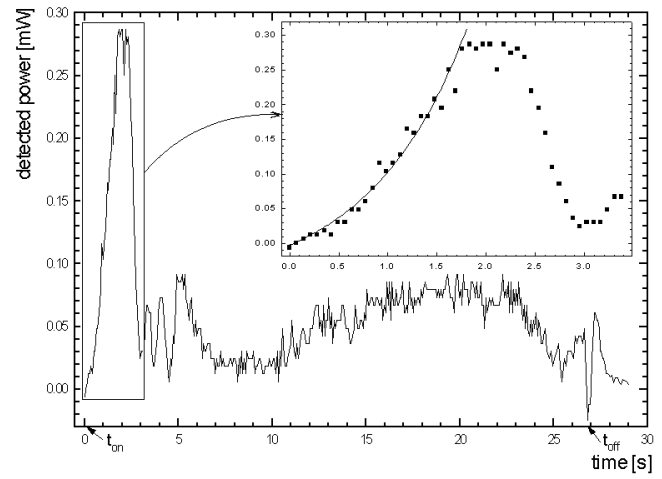

Fig. 3. Method of extrapolation of the nonlinear reorientation time from experimental data.

place of appearing of minimum interference ring (see Fig. 2b). The low-frequency electric field was obtained from the square function generator.

In Fig. 3 the response of the nematic layer on switching of the laser to the power of $51 \mathrm{~mW}$ for 27 seconds is presented. First, the optical field generated three minimum interference rings (compare with Fig. 4). The appearing of the fourth interference ring was blocked by existing electric field at $8.3 \mathrm{kV} / \mathrm{m}$ and in the end of measuring gate it disappeared. The dynamics is especially important in the initial reorientational process, therefore we take into account only the period of time needed to achieve the first maximum (zoomed frame in Fig. 3). The data in Fig. 3 was fitted by the exponential function $y=-0.06+0.06 \exp (t / \tau)$.

The inverted time $\tau$ dependence on square electric field for different values of input light power is shown in Fig. 5. The obtained inverse proportional relation of characteristics fully agrees with the theoretical prediction made in Eq. (4). The initial perturbations were a result of instabilities of generator, and they are not essential for making the trend of curve shape. 

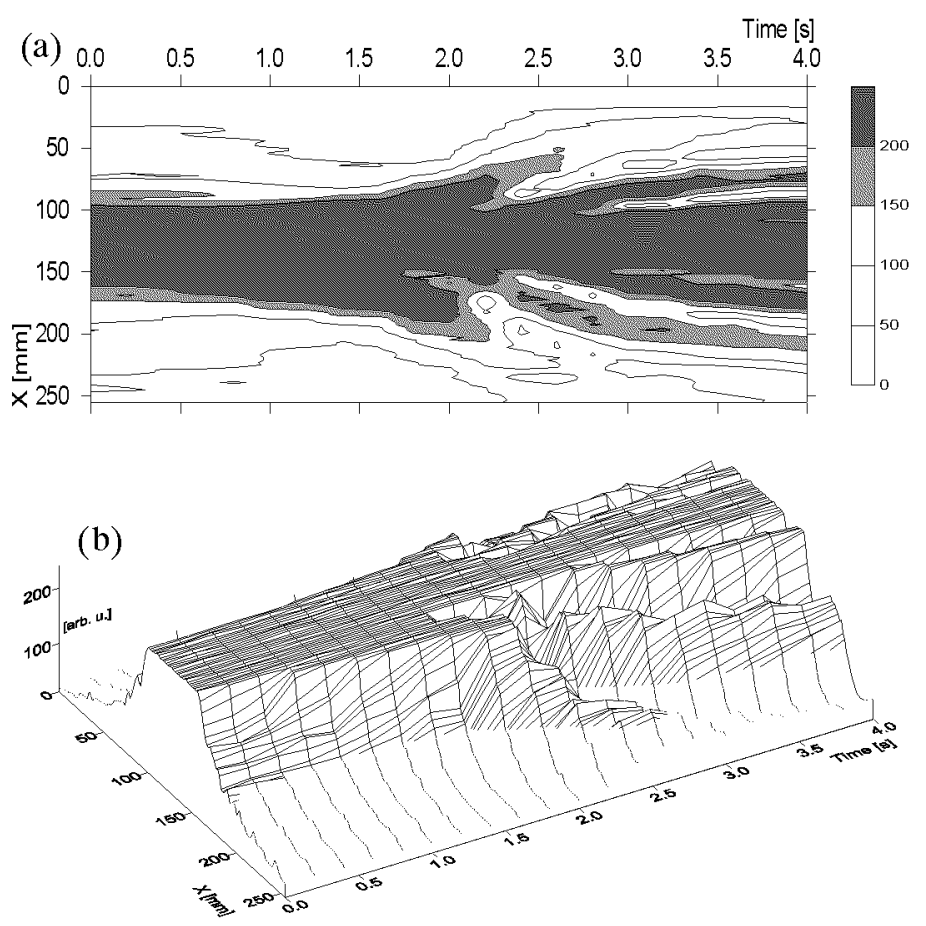

Fig. 4. Time dependence of output beam in diffraction image cross-section due to switching on the laser.

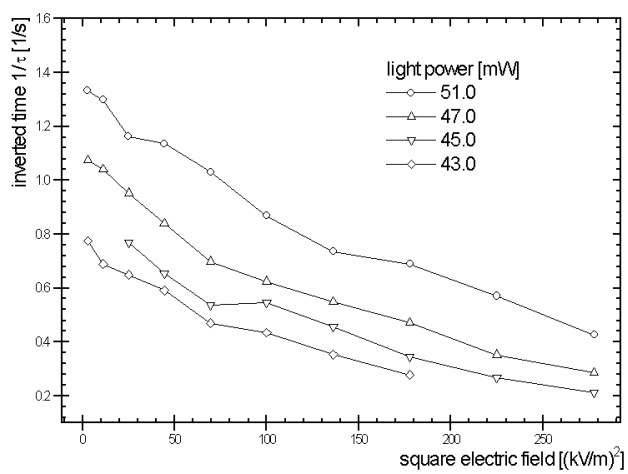

Fig. 5. Relation of inverted reorientation time vs. square electric field for different values of input light power.

The square optical field can be easy transformed into optical power, and the inverted time $\tau$ should be proportional to the power. The results presented in Fig. 6 confirm this relation. The influence of the small changes of input light power and value of the applied electric field on observable appearing and disappearing of new minimum interference rings is presented in Fig. 7. The number of visible 


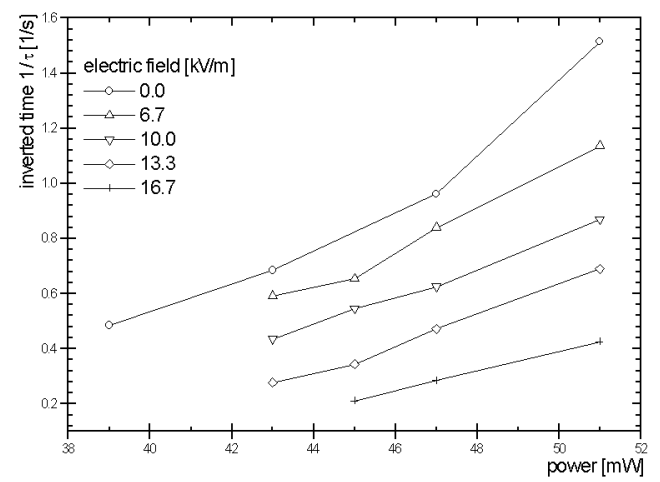

Fig. 6. Relation of inverted reorientation time vs. input light power for different values of square electric field.

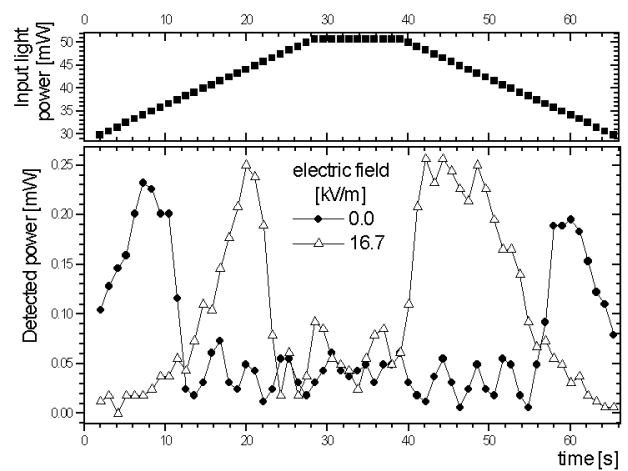

Fig. 7. Influence of small changes of input light power and value of applied electric field on observable appearing and disappearing of new minimum interference rings.

minimum rings decreases rapidly if we put electric field strong enough or the input laser power too small.

\section{Conclusions}

It should be noted that we measured time of appearance of the interference rings, which is not exactly the relaxation time described by Eq. (4). However, the existence of rings is determined by the phase difference between the center and the edge of light beam outcoming from the nematic layer. This means that the measured time is roughly equal to the relaxation time defined by Eq. (4) in the beam center $\tau(r=0)$.

The obtained results show that the measured time is dependent on the electric and optical field as defined by relation (4). By using these relations it is possible to measure material constants in nematic liquid crystals: elastic constant $K$ and 
rotational viscosity $\gamma$. The measured values are in good agreement with measurements obtained by other methods. It seems that the proposed method gives better results than the methods based on determination of the threshold power.

In our experiments the low-frequency electric field suppresses the reorientation. As a result the increase in the applied field intensity causes increase in the time of appearance of the nonlinear effect. However, if the low-frequency electric field supports the reorientation the relaxation time will be reduced. Such method of increasing the speed of nonlinear effect can be applied for example in nematics with negative electrical anisotropy.

\section{Acknowledgments}

This work was partially supported by the State Committee for Scientific Research under the grant No. 4 T11B 02622.

\section{References}

[1] N.V. Tabiryan, A.V. Sukhov, B.Ya. Zeldovich, Mol. Cryst. Liq. Cryst. 136, 1 (1986).

[2] I.C. Khoo, S.T. Wu, Optics and Nonlinear Optics of Liquid Crystals, World Scientific, Singapore 1993.

[3] I.C. Khoo, T.H. Liu, P.Y. Yan, J. Opt. Soc. Am. B 4, 115 (1987).

[4] W.K. Bajdecki, M.A. Karpierz, Acta Phys. Pol. A 95, 793 (1999).

[5] H.L. Ong, Phys. Rev. A 28, 2393 (1983).

[6] W.K. Bajdecki, M.A. Karpierz, in: Proc. XIV Conf. on Liquid Crystals: Chemistry, Physics, and Applications, Eds. J. Rutkowska, S.J. Klosowicz, J. Zielinsky, Proc. SPIE, Vol. 4759, 2002, p. 350. 